\title{
Proportional Assist Ventilation Improves Exercise Capacity in Patients with Obesity
}

\author{
Michael Dreher Hans-Joachim Kabitz Verena Burgardt Stephan Walterspacher \\ Wolfram Windisch \\ Department of Pneumology, University Hospital Freiburg, Freiburg, Germany
}

\section{Key Words}

Noninvasive ventilation · Obesity · Dyspnea $\cdot$ Proportional assist ventilation

\begin{abstract}
Background: Exercise capacity is reduced in obese patients due to disadvantageous respiratory mechanics that lead to dyspnea. Proportional assist ventilation (PAV) has the potential to unload resistive and elastic burdens of the ventilatory system. Objectives: The present study aimed to test if PAV can increase endurance and reduce exercise-related dyspnea in obese patients. Methods: Two symptom-limited exercise tests were performed at $75 \%$ of $\dot{V}_{2 m a x}$ : (1) without PAV and (2) with PAV. Exercise endurance, blood gases, dyspnea and limb discomfort (Borg scale) were assessed. Responders to PAV were defined as those increasing their exercise duration by more than 20\%. Results: Eighteen male obese patients (body mass index $35 \pm 5 \mathrm{~kg} / \mathrm{m}^{2}$ ) were investigated. Overall, PAV increased exercise duration by $13 \%(p=0.037)$ and improved exercise-induced dyspnea ( $p=0.004)$. In responders $(n=10)$, PAV increased the exercise duration by $31 \%$ from $721 \pm 300$ to $1,041 \pm 454 \mathrm{~s}(\mathrm{p}<0.001)$ and reduced dyspnea ( $p=0.004$ ) as well as limb discomfort ( $p=0.016$ ). Following stepwise multiple linear regression analysis, prolonged exercise time with PAV can only be predicted from total lung capacity $(p=0.02)$, considering total lung capacity,
\end{abstract}

$\dot{V}_{2 m a x}$ and body mass index. Conclusions: PAV applied during exercise led to an overall prolongation in exercise endurance and a reduction in dyspnea in obese patients. There was a broad spectrum of response to PAV, with more than $50 \%$ of patients increasing their exercise endurance by a mean of $31 \%$. Therefore, PAV might serve as a novel treatment option to enhance exercise capacity in a subgroup of obese patients in rehabilitation programs.

Copyright $\odot 2009$ S. Karger AG, Basel

\section{Introduction}

Obesity is highly prevalent in industrial countries (e.g., in 2003-2004, 17\% of children and adolescents in the United States of America were overweight and 32\% of adults were obese) [1] and challenges modern health systems by increasing the risk of all-cause mortality [2]. Even slight weight loss in obese individuals is sufficient to reduce cardiovascular risk factors such as arterial hypertension $[2,3]$. However, maintenance of weight loss is difficult, and the socioeconomic impact of obesity on health systems is high [2].

M.D. and H.-J.K. contributed equally to this work.

\section{KARGER}

Fax +41613061234 E-Mail karger@karger.ch www.karger.com
(C) 2009 S. Karger AG, Basel 0025-7931/10/0802-0106\$26.00/0

Accessible online at:

www.karger.com/res
Michael Dreher, MD

Department of Pneumology, University Hospital Freiburg

Killianstrasse 5, DE-79106 Freiburg (Germany)

Tel. +49761 270 3706, Fax +49761 2703704

E-Mail michael.dreher@uniklinik-freiburg.de 
Physical exercise in conjunction with an appropriate diet remains the most effective means of achieving and maintaining weight loss $[4,5]$. However, many obese, but otherwise healthy, patients are restrained during physical exercise due to increased body weight and exercise-induced dyspnea $[6,7]$.

Noninvasive ventilation is known to improve exercise capacity in patients with varying diseases, including chronic obstructive pulmonary disease [8-10] as well as restrictive thoracic disorders $[11,12]$. Specifically, proportional assist ventilation (PAV) is capable of unloading both the resistive and elastic burdens [13], which are thought to be high in patients with obesity. In addition, PAV generates inspiratory pressures which are dependent on the patient's inspiratory demand [13], thus making this mode of noninvasive ventilation particularly suitable for ventilation-aided physical activity. Accordingly, PAV has previously been shown to increase exercise capacity in healthy subjects [14] and chronic obstructive pulmonary disease patients [15-18].

Consequently, PAV might also have the potential to enhance exercise endurance and reduce dyspnea in patients with obesity. However, this has never been investigated before in clinical trials. Therefore, the present study aimed to evaluate the effectiveness of PAV application during exercise in obese patients. It was hypothesized that PAV is capable of enhancing exercise endurance and reducing exercise-induced dyspnea in these patients. If so, PAV could be considered as a novel treatment option to improve exercise capacity in obese patients and thereby substantially support initiation and maintenance of weight loss.

\section{Methods}

The study protocol was approved by the Institutional Review Board for Human Studies at the Albert-Ludwigs University, Freiburg, Germany, and was performed in accordance with the ethical standards laid down in the Declaration of Helsinki. Written informed consent was obtained from all patients.

\section{Patients}

Male outpatients with obesity (body mass index, BMI $>30 \mathrm{~kg} /$ $\mathrm{m}^{2}$ ) were consecutively enrolled in the study. Exclusion criteria included airway obstruction (forced expiratory volume in $1 \mathrm{~s} /$ forced vital capacity $<70 \%$ ), neuromuscular diseases, orthopedic disorders reducing exercise ability, and untreated/uncontrolled arterial hypertension both at rest and during exercise. Restrictive ventilation disorders were not considered as exclusion criteria, since obesity is known to result in some degree of restrictive ventilatory disorder. However, other causes of restrictive ventilation disorder, such as restrictive thoracic disorders or restrictive lung disease, resulted in exclusion of the patient.
Lung Function and Exercise Testing

Lung function parameters (Masterlab-Compact ${ }^{\circledR}$ Labor, Jaeger, Hochberg, Germany) were assessed in accordance with international guidelines [19]. Blood gases were taken from the arterialized earlobe (cobas b221 ${ }^{\circledR}$, Roche, Stuttgart, Germany). Ratings of perceived exertion for dyspnea (BDS) and limb discomfort (BLDS) were measured using a modified Borg scale [20]. For exercise testing, a computer-controlled, eddy current brake cycle ergometer (Ergoselect $100{ }^{\circledR}$, Ergoline, Bitz, Germany) was used. Heart rate, gas exchange (modified breath-by-breath method) and ventilation were each registered by an ergospirometric device (ZAN$600^{\circledR}, \mathrm{ZAN}$, Oberthulba, Germany).

A standardized incremental workload test (90 s rest; 90 s unloaded pedaling; $180 \mathrm{~s}$ lasting 10 - to 20 -Watt steps until exhaustion) [21] was performed in each patient to asses the maximal oxygen uptake $\left(\dot{\mathrm{V}}_{2 \max }\right)$. Pedaling frequency was maintained at 60-65 rpm throughout the entire exercise protocol. Based on these data, symptom-limited exercise capacity was determined with a constant-load exercise test at $75 \%$ of the previously determined $\dot{\mathrm{VO}}_{2 \max }$ according to previous investigations [22, 23]. During symptom-limited exercise (dyspnea/limb discomfort), patients were instructed to proceed with the exercise until exhaustion. The exercise was terminated by a fall below the required pedaling frequency $(60 \mathrm{rpm})$ for 3 consecutive seconds. Standardized verbal encouragements were given every 2 min throughout the exercise protocol.

\section{Noninvasive Ventilation}

PAV (BiPAP ${ }^{\circledR}$ Vision, Ventilatory Support System, Respironics Inc., Murrysville, Pa., USA) was provided in all cases. A complete description of PAV can be found elsewhere [13, 24]. All patients used a commercially available full-face mask (Ultra Mirage Full Face NVTM, ResMed, San Diego, Calif., USA).

Patients were ventilated with a flow assist of $15 \mathrm{~cm} \mathrm{H}_{2} \mathrm{O} / \mathrm{l} / \mathrm{s}$, a volume assist of $4 \mathrm{~cm} \mathrm{H}_{2} \mathrm{O} / 1$ and a \% Set of $30 \%$. End-expiratory positive airway pressure was set at 4 mbar. Maximal inspiratory airway pressure delivered by PAV was set at 40 mbar and the maximal tidal volume was set at $4,000 \mathrm{ml}$. No patient received supplemental oxygen.

\section{Study Design}

Patients performed 3 exercise tests with at least $5 \mathrm{~h}$ of rest between each trial: a standardized incremental workload test to asses $\dot{V}_{2 \max }$, followed by 2 symptom-limited exercise tests: first, without PAV under spontaneous breathing, and second, with additional PAV (fig. 1). Before and immediately after each exercise test, blood gases and ratings of perceived exertion were assessed. Furthermore, exercise time was recorded. For post hoc analysis, patients were divided in 2 groups: those who increased their exercise time by more than $20 \%$ when exercise was performed with PAV ('responder') and those who did not ('non-responder').

\section{Predetermination of Study Endpoints}

Differences in time between PAV-aided and -unaided exercise served as the primary endpoint. Secondary endpoints were defined as differences in ratings of perceived exertion.

Statistical Analysis

Statistical analysis was performed using Sigma-Stat ${ }^{\circledR}$ (version 3.1, Systat Software, Inc., Point Richmond, Calif., USA). Unless 
Table 1. Demographic data, lung function parameters and blood gas analyses of all patients $(n=18)$ at baseline

\begin{tabular}{lc}
\hline Age, years & $41.4 \pm 7.0$ \\
BMI, kg/m & $34.8 \pm 4.8$ \\
Pack years & $16.4 \pm 14.8$ \\
FVC, \% pred. & $105 \pm 10$ \\
$\mathrm{FEV}_{1}, \%$ pred. & $101 \pm 14$ \\
$\mathrm{FEV}$ & /FVC, \% \\
$\mathrm{TLC}^{2} \%$ pred. & $78 \pm 5$ \\
$\mathrm{PaO}_{2}, \mathrm{~mm} \mathrm{Hg}$ & $91 \pm 13$ \\
$\mathrm{PaCO}_{2}, \mathrm{~mm} \mathrm{Hg}$ & $78 \pm 5$ \\
$\mathrm{HCO}_{3}^{-}, \mathrm{mmol} / \mathrm{l}$ & $38 \pm 2$ \\
\end{tabular}

$\mathrm{FVC}=$ Forced vital capacity; $\%$ pred. = percentage predicted from calculated normal value; $\mathrm{FEV}_{1}=$ forced expiratory volume in $1 \mathrm{~s} ; \mathrm{PaO}_{2}=$ arterial partial pressure of oxygen; $\mathrm{PaCO}_{2}=$ arterial partial pressure of carbon dioxide; $\mathrm{HCO}_{3}^{-}=$bicarbonate.

Table 2. Comparison of post-exercise blood gases and heart rate between exercise with and without PAV

\begin{tabular}{lllll}
\hline & $\begin{array}{l}\text { Without } \\
\text { PAV }\end{array}$ & $\begin{array}{l}\text { With } \\
\text { PAV }\end{array}$ & $\begin{array}{l}\text { 95\% CI for } \\
\text { the difference }\end{array}$ & $\begin{array}{l}\mathrm{p} \\
\text { value }\end{array}$ \\
\hline $\mathrm{PaO}_{2}, \mathrm{~mm} \mathrm{Hg}$ & $81.8 \pm 7.0$ & $84.0 \pm 8.4$ & -1.5 to 6.0 & 0.225 \\
$\mathrm{PaCO}_{2}, \mathrm{~mm} \mathrm{Hg}$ & $34.5 \pm 3.7$ & $34.9 \pm 3.7$ & -1.2 to 1.9 & 0.630 \\
$\mathrm{pH}$ & $7.37 \pm 0.04$ & $7.37 \pm 0.03$ & -0.012 to 0.013 & 0.927 \\
$\mathrm{HCO}_{3}^{-}, \mathrm{mmol} / 1$ & $19.4 \pm 2.9$ & $19.5 \pm 2.9$ & -0.5 to 0.9 & 0.581 \\
Heart rate/min & $150.7 \pm 21.5$ & $151.8 \pm 18.3$ & -3.1 to 5.3 & 0.584
\end{tabular}

$\mathrm{PaO}_{2}=$ Arterial partial pressure of oxygen; $\mathrm{PaCO}_{2}=$ arterial partial pressure of carbon dioxide; $\mathrm{HCO}_{3}^{-}=$bicarbonate.

stated otherwise, all data were presented as the mean \pm standard deviation after testing for normal distribution (KolmogorovSmirnov test). The null hypothesis $\left(\mathrm{H}_{0}\right)$ was defined as there being no difference in exercise time between exercise with and without PAV. A sample size determination (paired t-test, power 0.8, 2-sided type I error 0.05) was performed with an estimated standard deviation of $180 \mathrm{~s}$ for the mean difference of exercise endurance, and a difference of at least $130 \mathrm{~s}$ between exercise with and without PAV. At least 18 subjects were needed for $\mathrm{H}_{0}$ rejection. The paired $t$ test was used for the quantitative measurements, and the Wilcoxon signed-rank test was used for the BDS and BLDS. For normally distributed data, the $95 \%$ confidence interval of the mean (95\% CI) was given where appropriate. Two-group comparison (responders vs. non-responders) was performed using the unpaired t-test for normally distributed data. Linear regression analysis was performed where appropriate [25]. Statistical significance was assumed with a p value $<0.05$.

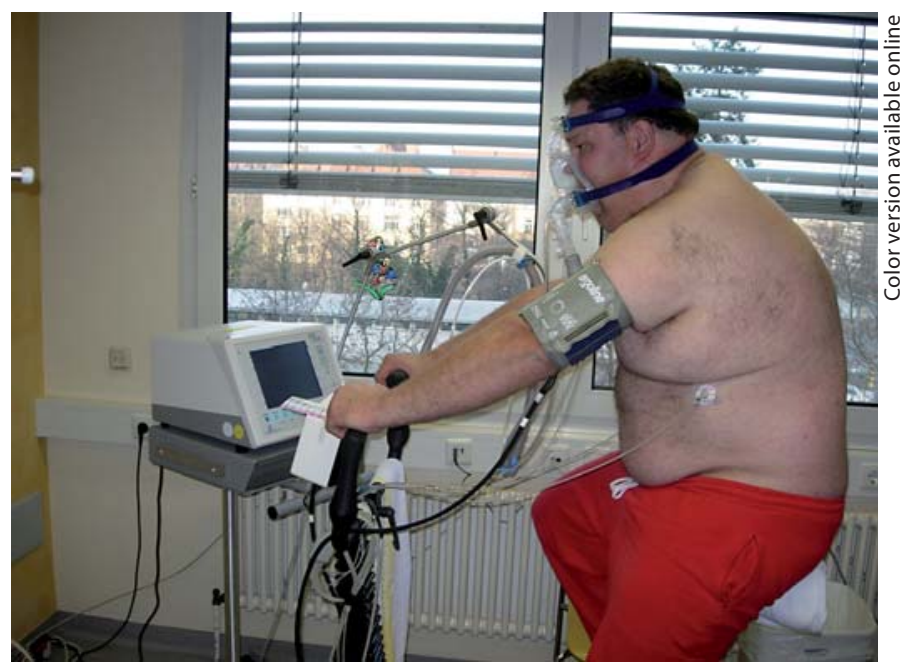

Fig. 1. Patient with obesity performing a symptom-limited incremental cycle exercise test with PAV.

\section{Results}

Eighteen male obese patients (BMI $34.8 \pm 4.8 \mathrm{~kg} / \mathrm{m}^{2}$ ), with demographic data shown in table 1 , were investigated. All patients were normocapnic and none of the investigated patients suffered from obesity hypoventilation syndrome. During the incremental workload test, the maximal work rate was $211 \pm 52 \mathrm{~W}$ and $\dot{\mathrm{VO}}_{2 \max }$ was 2.7 \pm 0.6 liters $/ \mathrm{min}$. When the symptom-limited exercise test was performed with PAV, exercise time increased by $13 \%$ to $1,069 \pm 460 \mathrm{~s}$ compared with $926 \pm 450 \mathrm{~s}(95 \% \mathrm{CI}$ $10-278 ; \mathrm{p}=0.037$; fig. 1 ) without PAV. In addition, after termination of exercise without PAV, the BDS was 3 (interquartile range 2-6), but this score was reduced to 2.5 (interquartile range $1-5 ; \mathrm{p}=0.004$ ) when exercise was performed with PAV. However, the BLDS after exercise was 5.5 (interquartile range 4-7) and 7 (interquartile range $4-8 ; \mathrm{p}=0.12$ ), respectively, indicating no difference between exercise with and without PAV. Furthermore, blood gases and heart rate after exercise did not differ between exercise with and without PAV (all p >0.05; table 2).

Ten out of 18 patients increased their exercise time by more than 20\% (responders). Responders increased their exercise time by $31 \%$ when the symptom-limited exercise test was performed with PAV; the exercise time was 721 $\pm 300 \mathrm{~s}$ without PAV and $1,041 \pm 454 \mathrm{~s}$ with PAV $(95 \%$ CI 193-450; $p<0.001$; fig. 2). In addition, the BDS of responders after exercise decreased from 4 (interquartile range $2-5$ ) to 2.5 (interquartile range $1-3 ; p=0.004$ ), and the BLDS of responders after exercise decreased from 7 


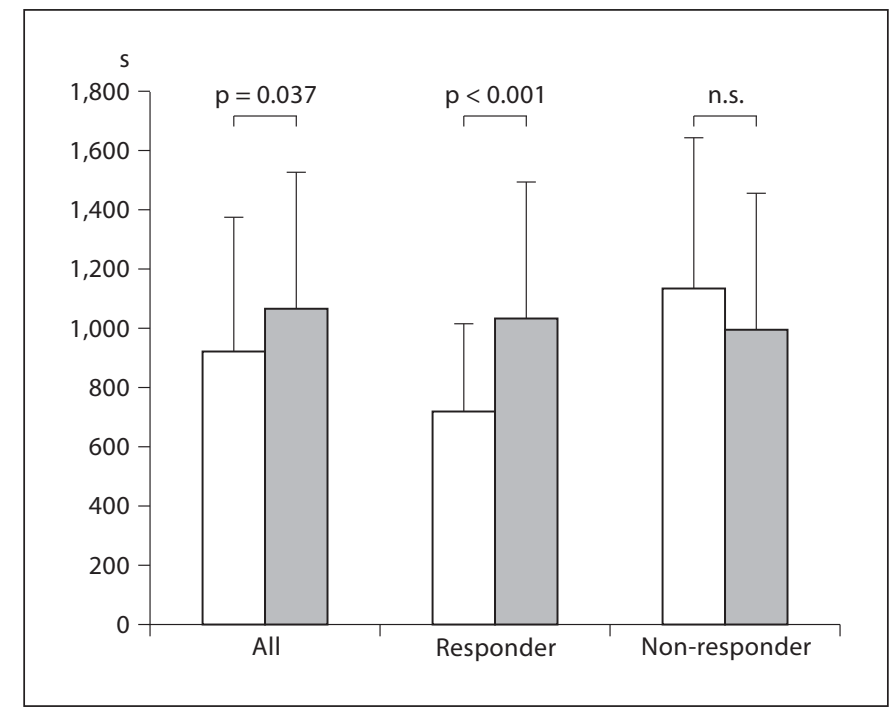

Fig. 2. Endurance time of all patients, responders $(\mathrm{n}=10)$ and non-responders $(\mathrm{n}=8)$, during symptom-limited incremental cycle exercise test with (grey bars) or without (white bars) PAV.
Table 3. Comparison of demographic data, lung function parameters and blood gas analyses between responders $(n=10)$ and nonresponders $(n=8)$ at baseline

\begin{tabular}{|c|c|c|c|c|}
\hline & Responders & $\begin{array}{l}\text { Non- } \\
\text { responders }\end{array}$ & $\begin{array}{l}95 \% \text { CI for } \\
\text { the difference }\end{array}$ & $\begin{array}{l}\mathrm{p} \\
\text { value }\end{array}$ \\
\hline Age, years & $42.1 \pm 5.4$ & $40.6 \pm 9.0$ & -8.7 to 5.7 & 0.67 \\
\hline Median height, $\mathrm{m}$ & 1.74 & 1.80 & & 0.056 \\
\hline Weight, kg & $110 \pm 18$ & $106 \pm 9$ & -19 to 10 & 0.526 \\
\hline BMI & $36.5 \pm 5.7$ & $32.7 \pm 2.0$ & -8.3 to 0.7 & 0.094 \\
\hline $\mathrm{FEV}_{1}, \%$ pred. & $96.5 \pm 11.9$ & $107.4 \pm 14.2$ & -2.1 to 24.0 & 0.093 \\
\hline FVC, \% pred. & $101.2 \pm 9.0$ & $111.0 \pm 7.3$ & $1.5-18.1$ & 0.024 \\
\hline TLC, \% pred. & $85.3 \pm 12.5$ & $97.1 \pm 11.0$ & -0.2 to 23.7 & 0.053 \\
\hline $\mathrm{PaO}_{2}, \mathrm{~mm} \mathrm{Hg}$ & $76.9 \pm 5.0$ & $80.0 \pm 4.4$ & -1.6 to 7.9 & 0.179 \\
\hline $\mathrm{PaCO}_{2}, \mathrm{~mm} \mathrm{Hg}$ & $39.0 \pm 2.0$ & $36.6 \pm 2.3$ & -4.6 to -0.2 & 0.032 \\
\hline $\mathrm{HCO}_{3}^{-}, \mathrm{mmol} / \mathrm{l}$ & $24.2 \pm 1.0$ & $22.9 \pm 1.2$ & -2.4 to -0.3 & 0.018 \\
\hline
\end{tabular}

$\mathrm{FEV}_{1}=$ Forced expiratory volume in $1 \mathrm{~s} ; \%$ pred. $=$ percentage predict ed from calculated normal value; $\mathrm{FVC}=$ forced vital capacity; $\mathrm{PaO}_{2}=$ arterial partial pressure of oxygen; $\mathrm{PaCO}_{2}=$ arterial partial pressure of carbon dioxide; $\mathrm{HCO}_{3}^{-}=$bicarbonate. (interquartile range 5-9) to 4.5 (interquartile range 4-7; $\mathrm{p}=0.016$ ) in favor of exercise with PAV. Blood gases and heart rate after exercise did not differ between exercise with and without PAV in responders (all $\mathrm{p}>0.05$ ).

Arterial partial pressure of carbon dioxide after the standardized incremental workload test was higher in responders compared with non-responders $(39.5 \pm 3.2$ vs. $34.0 \pm 3.7 \mathrm{~mm} \mathrm{Hg}$; 95\% CI 2.1-9.0; $\mathrm{p}=0.004)$. In addition, bicarbonate after exercise was higher in responders compared with non-responders $(20.0 \pm 2.4$ vs. $16.4 \pm 1.4$ $\mathrm{mmol} / \mathrm{l}$; 95\% CI 1.5-5.6; $\mathrm{p}=0.002$ ). Comparisons of baseline characteristics between responders and non-responders are shown in table 3.

Stepwise multiple linear regression analysis was performed to determine factors that may contribute to the improvement in exercise time with PAV. Considering 3 variables, total lung capacity (TLC; $\mathrm{p}=0.02), \mathrm{BMI}(\mathrm{p}=$ $0.26)$ and $\dot{\mathrm{V}}_{2 \max }(\mathrm{p}=0.06)$, the only factor significantly contributing to the improvement in exercise endurance was TLC.

\section{Discussion}

The present study shows that (1) the application of PAV during exercise is feasible in obese patients, and (2) PAV increased exercise endurance in obese patients. This is of potential clinical importance, since physical exercise is essential to both achieve and maintain weight loss in these patients $[4,5]$. For this reason, the use of PAV as a supplement during exercise training could act as a suitable adjunct in rehabilitation programs for patients with obesity.

Patients with obesity suffer from dyspnea even during mild exertion [26-28], which limits exercise capacity [6, 29]. In addition, recent data suggest that dyspnea at exertion is even prevalent in otherwise healthy obese patients due to the increased oxygen cost of breathing [30]; this has been postulated to account for the total energy required by respiratory muscles to overcome airway resistance, lung compliance, chest wall resistance, breathing inertia, antagonistic activity of respiratory muscles, chest wall distortion, gas compressibility and work on the abdominal viscera [30,31]. These respiratory mechanics are detrimentally altered in obese patients with low lung volume breathing, expiratory flow limitation, increased pulmonary resistance and decreased chest wall compliance [30], thus increasing the work of breathing [7]. However, a clear relationship between obesity-related alterations in respiratory mechanics and dyspnea has not yet been established [30, 32-34].

The present study has clearly shown that exercise-related dyspnea can be reduced by PAV. Since PAV is capable of unloading the resistive (by means of flow assist) and the elastic (by means of volume assist) burdens of the ventilatory system [13], the current authors suggest that 
PAV reduces the oxygen cost of breathing (although not shown), which has previously been linked to dyspnea [30]. Therefore, PAV can be regarded as a means of unloading respiratory muscles and reducing the work of breathing [35], leading to a subsequent decrease in the oxygen cost of breathing and, in turn, a reduction in exercise-related dyspnea. The overall effect is an increase in exercise endurance in obese patients. However, the link between PAV application and reduced oxygen cost of breathing needs to be confirmed.

In the present study, only 56\% (10 out of 18 ) patients responded positively to PAV according to predefined criteria. However, these responders demonstrated an increase of over $30 \%$ in exercise time and showed improvements in dyspnea and limb discomfort during exertion. In addition, lung volumes were lower in responders, and this is in line with previous findings in patients with restrictive thoracic disorders [12]. Furthermore, only TLC, but not $\mathrm{BMI}$ or $\dot{\mathrm{VO}}_{2 \max }$, was predictive of the enhanced exercise capacity with PAV. Therefore, the present study suggests that obese patients with reduced TLC are likely to be the subgroup to benefit most from exercise-assisted PAV. While this is consistent with the earlier work of Borel et al. [12] in patients with restrictive chest wall disorders, further work in obese patients is needed to confirm the current findings.

The present study has some limitations which should be addressed. First, randomization of the 2 study arms (exercise with vs. without PAV) was not performed, but BLDS reached maximal values during exercise without PAV, excluding a learning effect. In addition, an increase in exercise endurance by more than $30 \%$ can hardly be attributed to a learning effect. Secondly, flow assist and volume assist of PAV were not adjusted individually; instead, a predefined setting was chosen. Therefore, refine- ment of ventilatory strategies might even further improve exercise endurance in obese patients. Thirdly, it might be argued that the post hoc analysis was subject to bias, and the sample size is small. However, it further supports the results of the regression analysis by showing that impairments of lung function parameters might play a key role in predicting who might respond from PAV and who might not.

Furthermore, it must be mentioned that training of obese patients with PAV implies relatively high organizational and financial expenditures. However, good training results can only be achieved with a highly motivated patient, who in particular is willing to further sustain physical activity at home. Therefore, motivation of the patient to perform physical activity at home seems to be one of the major goals in these patients.

In conclusion, PAV applied during exercise led to an overall prolongation in exercise endurance and reduced dyspnea in obese patients. There was a broad spectrum of response to $\mathrm{PAV}$, and while some patients did not benefit at all, more than $50 \%$ of patients increased their exercise endurance by a mean of $31 \%$. Furthermore, the present study has shown that obese patients with reduced TLC are those who benefited most from PAV. Therefore, PAV might serve as a novel treatment option to enhance exercise capacity in a subgroup of obese patients.

\section{Acknowledgements}

All patients are gratefully acknowledged for their effort devoted to this study. We thank Dr. Sandra Dieni for her helpful comments on the manuscript prior to submission. The study group received an open research grant from Breas Medical AB, Molnlycke, Sweden, and from Respironics Inc., Pittsburgh, Pa., USA.

\section{References}

1 Ogden CL, Carroll MD, Curtin LR, McDowell MA, Tabak CJ, Flegal KM: Prevalence of overweight and obesity in the United States, 1999-2004. JAMA 2006;295:1549-1555.

\2 Ogden CL, Yanovski SZ, Carroll MD, Flegal KM: The epidemiology of obesity. Gastroenterology 2007;132:2087-2102.

-3 Stevens VJ, Obarzanek E, Cook NR, Lee IM, Appel LJ, Smith West D, Milas NC, Mattfeldt-Beman M, Belden L, Bragg C, Millstone M, Raczynski J, Brewer A, Singh B, Cohen J: Long-term weight loss and changes in blood pressure: results of the Trials of Hypertension Prevention, phase II. Ann Intern Med 2001;134:1-11.

\footnotetext{
4 Pearce PZ: Exercise is medicine. Curr Sports Med Rep 2008;7:171-175.

5 McMurray RG, Harrell JS, Creighton D, Wang Z, Bangdiwala SI: Influence of physical activity on change in weight status as children become adolescents. Int J Pediatr Obes 2008;3:69-77.

6 Whipp BJ, Davis JA: The ventilatory stress of exercise in obesity. Am Rev Respir Dis 1984; 129:S90-S92.

7 Parameswaran K, Todd DC, Soth M: Altered respiratory physiology in obesity. Can Respir J 2006;13:203-210. acute effects of noninvasive ventilatory support during exercise on exercise endurance and dyspnea in patients with chronic obstructive pulmonary disease: a systematic review. J Cardiopulm Rehabil 2002;22:290297.

9 Ambrosino N, Strambi S: New strategies to improve exercise tolerance in chronic obstructive pulmonary disease. Eur Respir J 2004;24:313-322.

10 Dreher M, Storre JH, Windisch W: Noninvasive ventilation during walking in patients with severe COPD: a randomised cross-over trial. Eur Respir J 2007;29:930-936.
} 
11 Tsuboi T, Ohi M, Chin K, Hirata H, Otsuka N, Kita H, Kuno K: Ventilatory support during exercise in patients with pulmonary tuberculosis sequelae. Chest 1997;112:10001007.

12 Borel J, Wuyam B, Chouri-Pontarollo N, Deschaux C, Levy P, Pepin J: During exercise non-invasive ventilation in chronic restrictive respiratory failure. Respir Med 2008; 102:711-719.

13 Younes M: Proportional assist ventilation, a new approach to ventilatory support. Theory. Am Rev Respir Dis 1992;145:114-120.

- 14 Babcock MA, Pegelow DF, Harms CA, Dempsey JA: Effects of respiratory muscle unloading on exercise-induced diaphragm fatigue. J Appl Physiol 2002;93:201-206.

15 Dolmage TE, Goldstein RS: Proportional assist ventilation and exercise tolerance in subjects with COPD. Chest 1997;111:948-954.

- 16 Bianchi L, Foglio K, Pagani M, Vitacca M, Rossi A, Ambrosino N: Effects of proportional assist ventilation on exercise tolerance in COPD patients with chronic hypercapnia. Eur Respir J 1998;11:422-427.

17 Hernandez P, Maltais F, Gursahaney A, Leblanc P, Gottfried SB: Proportional assist ventilation may improve exercise performance in severe chronic obstructive pulmonary disease. J Cardiopulm Rehabil 2001;21:135142.

18 Hawkins P, Johnson LC, Nikoletou D, Hamnegard C, Sherwood R, Polkey MI, Moxham $\mathrm{J}$ : Proportional assist ventilation as an aid to exercise training in severe chronic obstructive pulmonary disease. Thorax 2002;57: 853-859.
19 Quanjer PH, Tammeling GJ, Cotes JE, Pedersen OF, Peslin R, Yernault JC: Lung volumes and forced ventilatory flows. Report Working Party Standardization of Lung Function Tests, European Community for Steel and Coal. Official Statement of the European Respiratory Society. Eur Respir J Suppl 1993;16:5-40.

20 Borg GA: Psychophysical bases of perceived exertion. Med Sci Sports Exerc 1982;14:377381.

21 ATS/ACCP Statement on cardiopulmonary exercise testing. Am J Respir Crit Care Med 2003; 167:211-277.

22 van ' $t$ Hul A, Gosselink R, Hollander P, Postmus P, Kwakkel G: Acute effects of inspiratory pressure support during exercise in patients with COPD. Eur Respir J 2004;23: $34-40$.

-23 van 't Hul A, Gosselink R, Hollander P, Postmus P, Kwakkel G: Training with inspiratory pressure support in patients with severe COPD. Eur Respir J 2006;27:65-72.

24 Younes M, Puddy A, Roberts D, Light RB, Quesada A, Taylor K, Oppenheimer L, Cramp H: Proportional assist ventilation. Results of an initial clinical trial. Am Rev Respir Dis 1992;145:121-129.

25 Bland JM, Altman DJ: Regression analysis. Lancet 1986;i:908-909.

-26 Lawrence VJ, Kopelman PG: Medical consequences of obesity. Clin Dermatol 2004;22: 296-302.

27 Sahebjami H: Dyspnea in obese healthy men Chest 1998;114:1373-1377.

28 Gibson GJ: Obesity, respiratory function and breathlessness. Thorax 2000;55(suppl 1): S41-S44.
29 Sin DD, Jones RL, Man SFP: Obesity is a risk factor for dyspnea but not for airflow obstruction. Arch Intern Med 2002;162:14771481.

30 Babb TG, Ranasinghe KG, Comeau LA, Semon TL, Schwartz B: Dyspnea on exertion in obese women: association with an increased oxygen cost of breathing. Am J Respir Crit Care Med 2008;178:116-123.

31 Milic-Emili J, Orzalesi MM: Mechanical work of breathing during maximal voluntary ventilation. J Appl Physiol 1998;85:254258.

32 Babb TG, Buskirk ER, Hodgson JL: Exercise end-expiratory lung volumes in lean and moderately obese women. Int J Obes 1989;13: 11-19.

33 Babb TG, DeLorey DS, Wyrick BL, Gardner PP: Mild obesity does not limit change in end-expiratory lung volume during cycling in young women. J Appl Physiol 2002;92: 2483-2490.

34 DeLorey DS, Wyrick BL, Babb TG: Mild-tomoderate obesity: implications for respiratory mechanics at rest and during exercise in young men. Int J Obes (Lond) 2005;29:10391047.

-35 Kleinsasser A, Von Goedecke A, Hoermann C, Maier S, Schaefer A, Keller C, Loeckinger A: Proportional assist ventilation reduces the work of breathing during exercise at moderate altitude. High Alt Med Biol 2004; 5:420-428. 